\title{
Conservative versus Surgical Management of Proximal Humerus Fractures
}

\author{
Dr.Vishal Anand ${ }^{1}$, Dr.Ganesan Ganesan Ram ${ }^{2}$ \\ ${ }^{1,2}$ Department of orthopaedics, Sri Ramachandra Medical Collage, Tamilnadu.
}

\begin{abstract}
Prospective study of 26 patients of proximal humeral fracture admitted in the Department of Orthopaedics between June 2011 to December 2013 whether treated by conservative or surgical method were included in the study. Patients were evaluated for functional result by using The Swanson Shoulder Score and Constant Scoring System (Modified). Final outcome as per Swanson's Shoulder score and modified Constant score was maximum in surgically managed group. With good success rate the indications of operative treatment must be adhered to. The successful results can be attributed to early surgery, good preoperative planning, minimal soft tissue dissection, stable reduction; supervised postoperative exercise and regular follow up.
\end{abstract}

Keywords: Proximal humerus, Plating, Conservative, Shoulder score, Neer's classification.

\section{Introduction}

Proximal humeral fractures account for nearly 5\% of all fractures and are the third most common fracture after hip and distal radial fractures. It consists of $2 \%$ to $3 \%$ of upper extremity fractures, incidence to be about 73 per 100,000 populations with three fourths occurring after the age of 60 years and women out numbering men [1]. Fractures of proximal humerus account for 30\% to $40 \%$ of all humeral fractures. Treatment of proximal humeral fractures has been the subject of much controversy and confusion This is because of the complexity of these injuries, fracture displacement are difficult to see without careful radiographic views and associated soft tissue injuries. Further, there has always been diversity of opinion about the care of shoulder fractures, with frequent controversies and lively debate, further more even good anatomical results achieved at operative repair lead to poor results unless there is meticulous post operative rehabilitation, which can be more challenging in the shoulder than operative technique[2,3]. In this article we are going to discuss about conservative versus surgical management of proximal humerus fractures.

\section{Aim}

To compare the results of proximal humeral fractures treated surgically and conservatively.

\section{Materials And Methods}

Prospective study of 26 patients of proximal humeral fractures admitted in the Department of Orthopaedics between June 2011 to December 2013 whether treated by conservative or surgical method were included in the study. The inclusion criteria were Patients above 16 years of age, duration of injury less than 2 weeks, and Neer's two part, three part and four part fractures and Fracture dislocation. The exclusion criteria were any medical or surgical illness that may interfere with surgical procedure and anaesthesia, previous fractures of involved shoulder or any other shoulder pathology and history of previous neuromuscular weakness. Each case included in the study was evaluated clinically and radiologically. To study the type of injury the Neer's trauma series X-rays were done, which include (a) AP view and (b) Axillary view. The indication for Surgery were severely displaced two part fractures not reducible by close methods, Displaced three fragment factures with rotational displacement of articular fragment, Displaced four fragment fractures with malalignment and Fractures dislocation. Synthes Proximal humerus locking plate was used for all patients. For conservative management the most important criteria were examination of the proximal part of the humerus which could be moved with most of the motion occurring at the glenohumeral joint and not at the fracture site. All fractures were considered stable during the range of motion of the shoulder tolerated by patient. The involved extremity was immobilized in an arm to chest bandage or shoulder immobilizer for relief of pain. At the 3 weeks supervised active and assisted physiotherapy of shoulder was started under the guidance of physiotherapist on the OPD basis in the form of circumduction, wall clumping, rope pulling, back wiping, external rotation and pendulum exercises. The exercises for the shoulder were started with the patient in the supine position for forward elevation, external rotation and internal rotation. The patient was asked to follow up in 4 parts: 3 weeks, 6 weeks, 12 weeks and 18 weeks. Patients were evaluated for functional result by using The Swanson Shoulder Score and Constant Scoring System (Modified). 
III. Results

Table 1- Functional outcome as per the Swanson shoulder score

\begin{tabular}{|l|l|l|l|l|}
\hline \multirow{2}{*}{} & \multicolumn{2}{|l|}{ Surgically Managed } & \multicolumn{2}{l|}{ Conservatively Managed } \\
\cline { 2 - 5 } & No & $\%$ & No & $\%$ \\
\hline Excellent & 04 & 30.8 & - & - \\
\hline Good & 04 & 30.8 & 02 & 15.4 \\
\hline Fair & 05 & 38.4 & 07 & 53.8 \\
\hline Poor & - & - & 04 & 30.8 \\
\hline
\end{tabular}

Table 2- Functional outcome as per the constant scoring system

\begin{tabular}{|l|l|l|l|l|}
\hline \multirow{2}{*}{} & \multicolumn{2}{|l|}{ Surgically Managed } & \multicolumn{2}{l|}{ Conservatively Managed } \\
\cline { 2 - 5 } & No & $\%$ & No & $\%$ \\
\hline Excellent & 02 & 15.4 & - & - \\
\hline Good & 02 & 15.4 & - & - \\
\hline Fair & 05 & 38.4 & 03 & 23.08 \\
\hline Poor & 04 & 30.8 & 10 & 76.92 \\
\hline
\end{tabular}

\section{Discussion}

Proximal humeral fractures constitute 4-5\% of all fractures of long bones. It constitute for 2-3\% of the fractures of upper limb. Incidence of these fractures are 73 per one lakh population and $75 \%$ of these fractures are seen in elderly. $80-85 \%$ of these fractures are amenable to conservative treatment, remaining $15-20 \%$ are significantly displaced and require some type of internal fixation. The average age incidence in our series of 26 patients analysed, ranging between 21 to 80 years was 50.80 years, which was consistent with the age incidence in studies done by Neer (55.3 years)[4,5] and in other studies the average age was 52 years[6]. Regarding sex incidence, study of literature reveals predominance of proximal humeral fractures in females in an elderly age group [7]. Studies also reveal that male to female ratio being 1:0.8, 1:1.3[8]. In our series the male to female ratio was 1.6:1, 16 among 26 patients were males. These fractures of proximal humerus have bimodal presentation with adolescents and younger middle age patients who are more prone for high velocity injuries most common among males forming one group and later these fractures are seen in elderly patients( $>50$ years) in which cases they are osteoporosis related.

There was predilection of the dominant side involvement in our study. In our series of 26 patients of proximal humeral fractures, the dominant to non-dominant side of involvement ratio was 2.25:1, 18 among 26 fractures were in the dominant side which was consistent with the involvement in a study showing a ratio of 1.5:1[9]. There was no bilateral involvement in any patient. The mode of injury commonly observed in our series was fall on ground accounting for 16(61.5\%) followed by road traffic accident which was $7(27 \%)$ and lastly other mode of injury which was $3(11.5 \%)$. These observations was found to be consistent with the study done by Herscovici et al. (2000) which revealed 20(50\%) history of fall, 19(45\%) road traffic accident and 01(5\%) others out of forty cases studied. In literature, it is said that the high energy injuries such as RTA are much less common than low energy domestic fall [10] but fast pace modern life, acceleration of travel increasing the number of fractures. In our series, the common mode of injury in relation to age observed was road traffic accident in 20-40 years of age group accounting for three out of six patients (50\%) and fall on ground in more elder groups i.e. 41-60 and >60 years age group accounting for nine out of fourteen (64.3\%) and six out of six (100\%) respectively. This is in consistent with a study done by Mohit G. (2010). Literature says fractures in adolescents and young adults are usually produced by high energy injuries especially from RTA, gunshot wounds, etc. but in elderly, it is usually due to low energy osteoporotic injuries specially form low energy domestic fall [10]. Neer's type II fracture was found to be the most common type of fractures in our study. Our series revealed $17(65 \%)$ were two part fractures, 06(23\%) were three part fractures and $03(12 \%)$ were four part fractures. It was found to be consistent with a study of 40 cases, where 20(50\%) were two part fractures, $16(40 \%)$ were three part fractures and $4(10 \%)$ were four part fractures. The study showed that functional recovery was good in two part fractures than more comminuted ones. In our series, fracture dislocation was found to be more common in three and four part proximal humeral fractures.

Final outcome as per Swanson's Shoulder score and modified Constant score was high in surgically managed group. Therefore, it was seen that the patients managed operatively showed better results than the patients managed conservatively. Results obtained in our study by surgical methods was compared and found to be consistent with the studies done by Plecko et al. (2005)[11] and David et al. (2009)[12] on management of proximal humeral fractures by plate showing mean constant score of 62.6 and 57.5 points respectively. Results obtained in our study by surgical methods was also compared and found to be better than the study done by Zyto (1998)[13] on the management of proximal humeral fractures by conservative method showing a mean constant score of 59 for three part and 47 for four part fractures of proximal humerus. 


\section{Conclusion}

Fracture of the proximal humerus is still a debatable and controversial subject in orthopaedics. Based on our experience and results we conclude that proximal humeral fractures were severely disabling inspite of surgeon best efforts. Full range of movement in severely comminuted fractures was an unlikely outcome. With good success rate the indications of operative treatment must be adhered to. The successful results can be attributed to early surgery, good preoperative planning, minimal soft tissue dissection, stable reduction; supervised postoperative exercise and regular follow up.

\section{References}

[1]. Court-Brown CM, Caesar B: Epidemiology of adult fractures: A review. Injury. 2006 Aug; 37(8): 691-7. Epub 2006 Jun 30.

[2]. Szyskowitz R, Seggl W, Schleifer P.Proximal humeral fractures management techniques and expected results. Clin-Orthop 1993: 292: 13-25.

[3]. Hawkins RJ, Kiefer Gin: Internal fixation for proximal humeral fractures. Clin-Orthop- 1987: 223:77-85

[4]. Neer C.S: Displaced proximal humeral fractures Part-I Classification and Evaluation JBJS (am) 1970: 52: $1077-89$.

[5]. Neer C.S: Displaced proximal humeral fractures Part-II Treatment of three and four part displacement .JBJS (am) 1970: 52: 10901101.

[6]. Herscovici, Dolfi Jr DO, Derrick's. DO, Saunders, Marie.P. LPN, Johnson, et al: Percutaneous fixation of proximal humeral fractures. Clin- Orthop 2000: 375: 97-104.

[7]. Steven.H. Rose Joseph Melton Bernard. F. Morrey et al. Epidemiological features of humeral fractures. Clin-Orthop-1982: 168: 2430 .

[8]. Kojy, Yamamoto R: Surgical treatment of complex fractures of the proximal humerus. Clin-Orthop- 1996: 327: $225-237$.

[9]. Nitin Jain, Ricardo Pietrobon, Shawn Hocker, Ulrich Guller, Anoop Shankar and Laurence D. Higgins. The Relationship between Surgeon and Hospital Volume and Outcomes of shoulder arthroplasty. J Bone Joint Surg Am. 2004; 86:496-505.

[10]. Bucholz and Heckman's Rockwood \& Green's Fractures in Adults. Vol-1: 7th Ed 2010, (Lippincott Williams and Wilkins Company, USA) Pg 1040.

[11]. Plecko M, Kraus A. Internal fixation of proximal humerus fractures using the locking proximal humerus plate. Operat Orthop Traumatol. 2005; 17(1):25-50.

[12]. David S. Thyagarajan, Samarth J. Haridas, Denise Jones, Colin Dent, Richard Evans and Rhys Williams. Functional outcome following proximal humeral interlocking system plating for displaced proximal humeral fractures. Int J Shoulder Surg. 2009 JulSep; 3(3): 57-62.

[13]. Zyto K. Non-operative treatment of comminuted fractures of the proximal humerus in elderly patients. Injury. 1998; 29(5):349-352. 